\title{
Excitonic properties of strained triple quantum-ring molecules
}

\author{
M. Tadić ${ }^{1,2, *}$ and F. M. Peeters ${ }^{1,3, \dagger}$ \\ ${ }^{1}$ Department of Physics, University of Antwerp, Groenenborgerlaan 171, B-2020 Antwerp, Belgium \\ ${ }^{2}$ Faculty of Electrical Engineering, University of Belgrade, P.O. Box 3554, 11120 Belgrade, Serbia \\ ${ }^{3}$ Departamento de Física, Universidade Federal do Ceara, Campus do Pici, Caixa Postal 6030, 60.455-760 Fortaleza, CE, Brazil
}

(Received 10 December 2008; published 20 April 2009)

\begin{abstract}
The tunneling coupling in three vertically stacked (In,Ga)As/GaAs quantum rings is investigated. With increasing inter-ring separation $(d)$, we find that the nonuniform strain results into a crossing of the lowestenergy electron states. Strain is also responsible for an increase in the ground electron energy above the level in the single quantum ring. The ground hole energy level exhibits decrease when $d$ decreases, which is typical for antibonding states in an unstrained structure. These effects lead to a local maximum in the dependence of the ground-state exciton energy on $d$. Our theoretical results compare well with recent photoluminescence measurements but deviate considerably from the calculations for flat bands in quantum-ring molecules. We conclude that the nonuniform character of the strain distribution gives rise to a peculiar exciton hybridization in self-assembled quantum-ring molecules.
\end{abstract}

DOI: 10.1103/PhysRevB.79.153305

The application of quantum-dot molecules as solid-state qubits triggered enormous interest in these artificial quantum systems. ${ }^{1,2}$ Several experiments analyzed exciton hybridization in vertical quantum-dot molecules by applying an electric field. ${ }^{3-5}$ It was shown that accurate modeling of the exciton states is necessary in order to describe the observed anticrossings of the energy levels. ${ }^{4,5}$ Ring-shaped quantum dots which are predicted to show a remarkable excitonic Aharonov-Bohm effect have been recently fabricated. ${ }^{6-8}$ These quantum rings were produced through a modification of the epitaxial growth conditions of self-assembled quantum dots. ${ }^{9}$ Also stacks of $(\mathrm{In}, \mathrm{Ga}) \mathrm{As} / \mathrm{GaAs}$ quantum rings, representing quantum-ring molecules, have been recently fabricated and the exciton energies were measured using photoluminescence. ${ }^{10}$ It was found that the individual rings in the quantum-ring molecule are almost perfectly aligned and have a narrow size distribution. ${ }^{10,11}$ Such high quality samples are favorable for applications in, e.g., quantum computing.

The exciton states in self-assembled quantum dots exhibit large binding energies due to confinement in all three spatial directions. They are affected by large strain, which is nonuniform inside the ring and penetrates in the matrix. It may lead to a complex hybridization between the single-particle and exciton states originating from the individual dots in quantum-dot molecules. As an example, Refs. 2 and 4 indicated that the energies of the exciton states in the (In,Ga)As/ GaAs quantum-dot molecules are blueshifted with respect to the values in the single quantum dot. Furthermore, the recent photoluminescence measurements on carefully prepared vertically coupled quantum-ring molecules seem to show a similar unusual behavior. ${ }^{10}$ The aim of the present Brief Report is to theoretically analyze the interplay between the nonuniform strain and tunneling coupling which is present in quantum-ring molecules.

We consider the system of three vertically coupled (In,Ga)As/GaAs quantum rings, similar to the one investigated in Ref. 10. Recently, exciton states in quantum-ring molecules were studied theoretically, ${ }^{12,13}$ but in these works strain, which is inherently present in self-assembled quantum rings, was neglected. Here, we will include and model the
PACS number(s): 73.21.La, 78.67.Hc

electron, hole, and exciton states for the experimental quantum-ring molecules of Ref. 10. The strain distribution is extracted from the continuum mechanical model. ${ }^{14}$ The electron and hole states are modeled by the single-band effective-mass theory. Its use relies on the dominant heavyhole envelope function in the Luttinger multiband spinor due to splitting between the effective potential wells for large compressive strain in the dot. ${ }^{4,5,15,16}$ The exciton states are computed using exact numerical diagonalization, where the exciton wave function is expanded in products of the electron and hole envelope functions. ${ }^{15}$ The analyzed rings are assumed to have the same size as the experimental ones. ${ }^{11}$

In the continuum mechanical model of strain, the elastic energy of the supercell of volume $V$, which is minimized, ${ }^{14}$ is given by

$$
\begin{aligned}
E_{\mathrm{el}}= & \int_{V} d V\left[\frac{1}{2} C_{11}\left(\varepsilon_{x x}^{2}+\varepsilon_{y y}^{2}+\varepsilon_{z z}^{2}\right)+C_{12}\left(\varepsilon_{x x} \varepsilon_{y y}+\varepsilon_{y y} \varepsilon_{z z}\right.\right. \\
& \left.+\varepsilon_{z z} \varepsilon_{x x}\right)+2 C_{44}\left(\varepsilon_{x y}^{2}+\varepsilon_{y z}^{2}+\varepsilon_{z x}^{2}\right)-2 \alpha\left(\varepsilon_{x x}+\varepsilon_{y y}+\varepsilon_{z z}\right) \varepsilon_{0} \\
& \left.+3 \alpha \varepsilon_{0}^{2}\right]
\end{aligned}
$$

where $\varepsilon_{i j}$ denotes the $i j$ component of the strain tensor $(i$ $=x, y, z), C_{i j}$ are the elastic constants in the reduced notation, and $\alpha=C_{11} / 2+C_{12} . \varepsilon_{0}$ denotes the misfit strain (lattice mismatch) between the dot and the barrier as follows:

$$
\varepsilon_{0}=\left(a_{0 r}-a_{0 m}\right) / a_{0 m},
$$

where $a_{0 r}$ and $a_{0 m}$ denote the lattice constant in the (In,Ga)As ring and the GaAs matrix, respectively. All components of the strain tensor are approximately axially symmetric, but full axially symmetry was imposed by averaging the three-dimensional (3D) strain field over the polar angle..$^{15}$ The finite element method is adopted to minimize the strain energy functional.

The single-particle effective-mass Schrödinger equation has the following form: 


$$
\begin{array}{r}
-\frac{\hbar^{2}}{2 m_{\|}} \frac{\partial^{2} \psi}{\partial \rho^{2}}-\frac{\hbar^{2}}{2 m_{\|}} \frac{1}{\rho} \frac{\partial \psi}{\partial \rho}+\frac{\hbar^{2}}{2 m_{\|}} \frac{l^{2}}{\rho^{2}} \psi-\frac{\hbar^{2}}{2} \frac{\partial}{\partial \rho}\left(\frac{1}{m_{\|}}\right) \frac{\partial \psi}{\partial \rho} \\
-\frac{\hbar^{2}}{2 m_{z}} \frac{\partial^{2} \psi}{\partial z^{2}}-\frac{\hbar^{2}}{2} \frac{\partial}{\partial z}\left(\frac{1}{m_{z}}\right) \frac{\partial \psi}{\partial z}+V_{\mathrm{eff}}(\rho, z) \psi=E \psi,
\end{array}
$$

where $\rho$ and $z$ are the cylindrical coordinates, $m_{\|}$and $m_{z}$ are the effective masses in the $x y$ plane and along the $z$ direction, respectively, $l$ denotes the single-particle orbital quantum number, $V_{\text {eff }}$ is the effective potential, $\psi$ the single-particle envelope function, and $E$ is the eigenenergy of the particle. $V_{\text {eff }}$ is given by

$$
V_{\text {eff }}(\rho, z)=V_{\text {off }}(\rho, z)+c_{\|}\left\langle\left(\varepsilon_{x x}+\varepsilon_{y y}\right)\right\rangle(\rho, z)+c_{z}\left\langle\varepsilon_{z z}\right\rangle(\rho, z) .
$$

Here $V_{\text {off }}$ is the confining potential due to the band offset and $c_{\|}$and $c_{z}$ are the relevant combinations of the electronic deformation potentials $a_{c}, a_{v}$, and $b$ (Ref. 17) given by

$$
c_{\|}=c_{z}=a_{c}
$$

for the electron in the conduction band and

$$
c_{\|}=-\left(a_{v}+\frac{b}{2}\right), c_{z}=-a_{v}+b
$$

for the heavy holes. The single-particle states are classified according to $l$, the principal quantum number $n$, and the parity $\sigma$, and are denoted by $n l^{\sigma}$.

Our exact diagonalization approach extracts the exciton states from the secular equation,

$$
\left(E_{e n}-E_{h m}-E_{\text {exc }}\right) \delta_{i k} \delta_{j m}+\sum_{k} \sum_{m}\left\langle\psi_{e i} \psi_{h j}\left|V_{C}\right| \psi_{e k} \psi_{h m}\right\rangle=0,
$$

where $V_{C}=-e^{2} /\left[4 \pi \epsilon \epsilon_{0}\left(\mathbf{r}_{\mathbf{e}}-\mathbf{r}_{\mathbf{h}}\right)\right]$ denotes the Coulomb potential energy between the electron and the hole, and $\psi_{e n}$ and $\psi_{h m}$ are the electron and hole wave functions, respectively. Here, the state is labeled by a single number, i.e., $i \equiv n_{e} l_{e i}$. A straightforward derivation gives

$$
\begin{aligned}
& \left\langle\psi_{e i} \psi_{h j}\left|V_{C}\right| \psi_{e k} \psi_{h m}\right\rangle \\
& =\frac{1}{4 \pi^{2}} \frac{e^{2}}{\epsilon \epsilon_{0}} \delta_{l_{e i}+l_{h j}=l_{e k}+l_{h m}} \int_{0}^{\infty} d k_{\|} k_{\|} \int_{-\infty}^{+\infty} d k_{z} \frac{1}{k_{\|}^{2}+k_{z}^{2}} \\
& \quad \times \mathcal{F}_{e}\left(k_{\|},-k_{z}\right) \mathcal{F}_{h}\left(k_{\|}, k_{z}\right),
\end{aligned}
$$

where $k_{\|}$and $k_{z}$ denote the in-plane and the $z$ component of the wave vector in Fourier space, respectively. $\mathcal{F}_{e}$ is the twodimensional transform given by

$$
\mathcal{F}_{e}\left(k_{\|}, k_{z}\right)=\int_{0}^{\infty} \rho d \rho \int_{-\infty}^{+\infty} d z \psi_{e i}^{*} \psi_{e k} J_{\left|l_{i}-l_{k}\right|}\left(k_{\|} \rho\right) e^{i k_{z} z} .
$$

$L=l_{e}+l_{h}$ is a good quantum number for the exciton as indicated by the Kronecker $\delta$ in Eq. (8). For a given $L$, the electron and hole states which satisfy $L=l_{e}+l_{h}$ only contribute to the exciton states. The other good quantum numbers of the exciton states are the principal quantum number $n$ and the parity of the exciton $\sigma_{\text {exc }}$. Therefore, the exciton states are labeled by $n L^{\sigma_{\text {exc }}}$.

For comparison purposes we consider also the case of
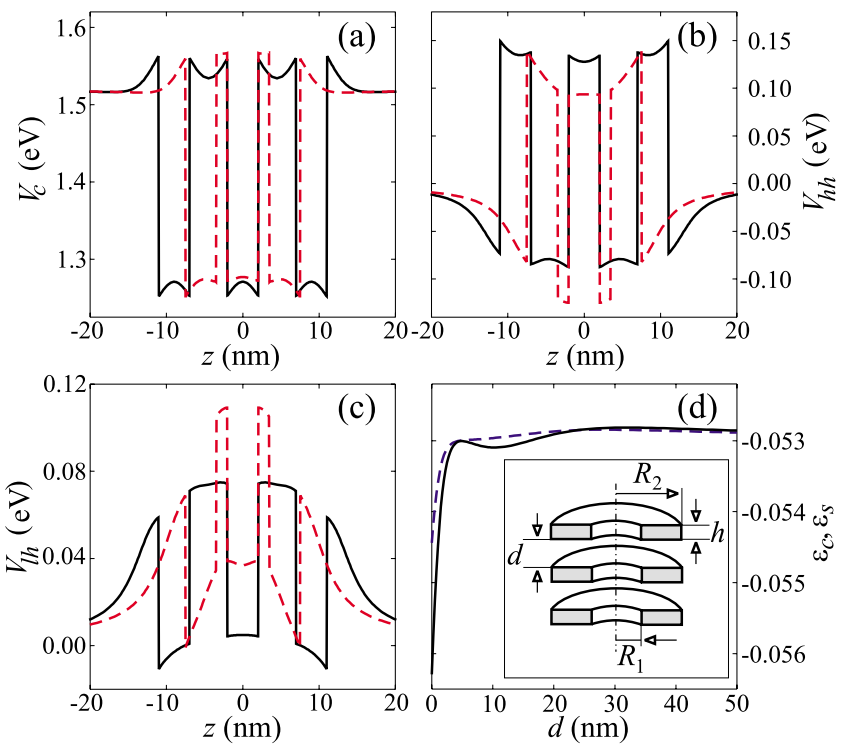

FIG. 1. (Color online) The effective potentials for $d=5 \mathrm{~nm}$ (solid lines) and $d=1.5 \mathrm{~nm}$ (dashed lines) for: (a) the electrons in the conduction band, (b) the heavy holes, and (c) the light holes. (d) Variation of the hydrostatic strain in the center of the central (solid line) and satellite rings (dashed line). The cross section of the rings is shown in the inset.

quantum-ring molecules where the strain is taken constant inside the ring,

$$
\varepsilon_{x x}=\varepsilon_{y y}=\frac{a_{0 m}-a_{0 r}}{a_{0 r}}, \quad \varepsilon_{z z}=-\frac{2 C_{12}}{C_{11}} \varepsilon_{x x},
$$

and zero in the matrix, as is valid for the case of quantum well structures.

A recent analysis using cross-sectional scanning tunneling microscopy (X-STM) (Ref. 11) indicated that the height $h$ of the fabricated (In,Ga)As/GaAs rings is about $4 \mathrm{~nm}$. Furthermore, the inner and outer radia of the ring were estimated to be $R_{1}=8 \mathrm{~nm}$ and $R_{2}=15 \mathrm{~nm}$, respectively. Therefore, the average radius of the analyzed ring $\langle R\rangle=\left(R_{1}+R_{2}\right) / 2$ equals $11.5 \mathrm{~nm}$. The alloy is taken to contain $55 \%$ of InAs in each ring as measured. The inter-ring separation $d$ is varied in the range from 0 to $50 \mathrm{~nm}$. All three quantum rings are assumed to be equal (homonuclear quantum-ring molecule) and to have a rectangular cross section, as shown in the inset of Fig. 1. The $s$ electron (hole) states have the lowest (highest) energy and are only analyzed here. The exciton wave functions are expanded in products of four electron and hole states for each $l$ in the range $[-7,7]$. Only the ground exciton $1 S^{+}$ state, which was experimentally measured in Ref. 10 is considered. The presence of the wetting layers is neglected in the calculation since they are generally only of importance for the higher states. The other material parameters in the model are the effective masses, the band offsets, the lattice constants, the elastic constants, and the deformation potentials in (In,Ga)As and GaAs, which are all taken from Ref. 18.

The single-particle wave functions are mainly localized inside the rings; therefore the effective potential for the electrons and holes shown in Figs. 1(a)-1(c) are plotted along the 




FIG. 2. (Color online) The variations in the electron even (solid lines) and odd (dashed line) energy levels with inter-ring distance. The upper inset shows a detail of this dependence on the range $d$ $\geq 10 \mathrm{~nm}$, while the lower inset shows the level splitting in the flat-band structure. The dotted line in the upper inset denotes the energy of the ground state in a single quantum ring.

direction $(\rho, z)=(\langle R\rangle, z)$, which passes through the rings. When the inter-ring distance decreases from $d=5 \mathrm{~nm}$ (solid line) to $d=1.5 \mathrm{~nm}$ (dashed line), the effective potential well for the electron becomes shallower inside the ring and the barrier height formed in the barrier between the dots increases. The effective potential for the heavy holes is similar to the one for the electrons [compare Figs. 1(a) and 1(b)], while the light holes are confined in the space between the two dots [see Fig. 1(c)], but the depth of the potential well is smaller than for the heavy holes.

In order to analyze the energy spectra into more detail, the hydrostatic strain $\varepsilon_{\text {hyd }}=\varepsilon_{x x}+\varepsilon_{y y}+\varepsilon_{z z}$ inside the central ring $\left[\varepsilon_{c}=\varepsilon_{\text {hyd }}(\langle R\rangle, 0)\right]$ and each of the satellite rings $\left\{\varepsilon_{s}\right.$ $\left.=\varepsilon_{\text {hyd }}[\langle R\rangle, \pm(d+h)]\right\}$ as it depends on $d$ is shown in Fig. 1(d). We point out that $a_{c}$ in Eq. (3) is negative; therefore a decrease in $\varepsilon_{c}$ and $\varepsilon_{s}$ implies that the effective potential well for the electron in the conduction band becomes shallower when $d$ decreases. The difference between $\varepsilon_{c}$ and $\varepsilon_{s}$ is not large, but the two curves in Fig. 1(d) cross at $d_{1}=24 \mathrm{~nm}$, and the effective potential wells in the upper and lower rings are deeper than the one in the central ring when $d$ increases beyond $d_{1}$.

The energies of the three lowest $l=0$ electron states in the conduction band $\left(1 s^{+}, 2 s^{+}\right.$, and $1 s^{-}$states) as function of $d$ are shown in Fig. 2, and the lower inset in this figure shows the dependence on the lowest $s$ states in case we use the approximation of a flat band inside the rings. Notice that the three levels in the flat-band quantum-ring molecules merge near half the value of $d$ as compared to the full nonuniform strain case. The effect arises from the strain variation with $d$, as illustrated in Fig. 1. Furthermore, we find a remarkable crossing of the $1 s^{+}$and $2 s^{+}$states in the strained structure around $d=22 \mathrm{~nm}$ (see the upper inset in Fig. 2). This value is close to the position of the crossing between $\varepsilon_{s}$ and $\varepsilon_{c}$ shown in Fig. 1(d). It effectively marks the transfer of the

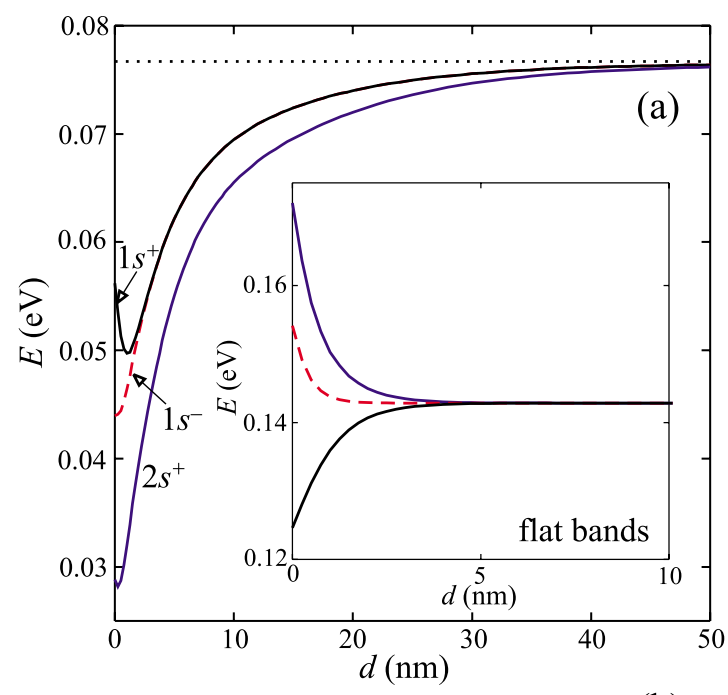

(b)

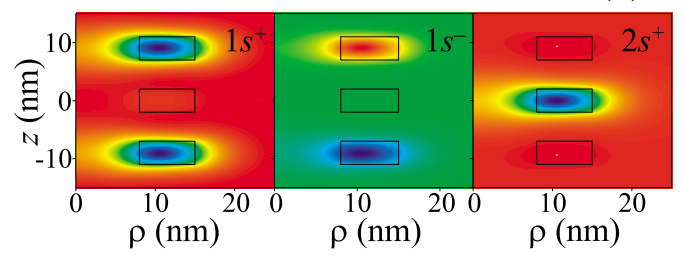

FIG. 3. (Color online) (a) The even (solid lines) and the odd (dashed line) hole levels as functions of the inter-ring separation. The eigenenergy in the single quantum ring is displayed as the horizontal dotted line. The inset shows the level splitting in the unstrained structure. (b) The wave functions of the $1 s^{+}, 1 s^{-}$, and $2 s^{+}$ states.

spatial localization of the electron eigenstates from the central ring for small $d$ to the upper and lower rings for large $d$. Furthermore, the convergence of the three lowest energies to the ground-state energy in the single quantum ring is rather slow in Fig. 2. Due to the slow decay of the effective potential in the quantum-ring molecules, the eigenenergies of the ground states in the quantum-ring molecule and the single quantum ring differ by $\Delta E=0.4 \mathrm{meV}$ at $d=50 \mathrm{~nm}$. On the other hand, $\Delta E$ is much smaller in the flat-band structure (it is less than $0.01 \mathrm{meV}$ even at $d=20 \mathrm{~nm}$ ). Hence, the effect of slow convergence of the eigenenergies arises essentially from the variation in the nonuniform strain field with $d$.

The $1 s^{+}, 1 s^{-}$, and $2 s^{+}$hole energy levels as function of $d$ are displayed in Fig. 3. Except for a small inter-ring distance $(d<2.5 \mathrm{~nm})$, the ground and the first-excited states are close to each other. Due to the presence of the nonuniform strain the effective potential wells in the upper and lower rings are deeper than the effective potential well in the central ring. Therefore, the wave functions of the $1 s^{+}$and $1 s^{-}$states are mainly localized inside the top and bottom rings, while the wave function of the $2 s^{+}$state is mainly localized in the central ring, as Fig. 3(b) demonstrates for $d=5 \mathrm{~nm}$. The eigenergies of all three displayed states increase continuously for $d>2 \mathrm{~nm}$, while a different trend is found in the flat-band structure, as shown in the inset of Fig. 3. Comparing Figs. 2 and 3, we might deduce that the effect of strain is larger on the hole states than on the electron states.

The energy of the $1 S^{+}$exciton state as a function of $d$ is 


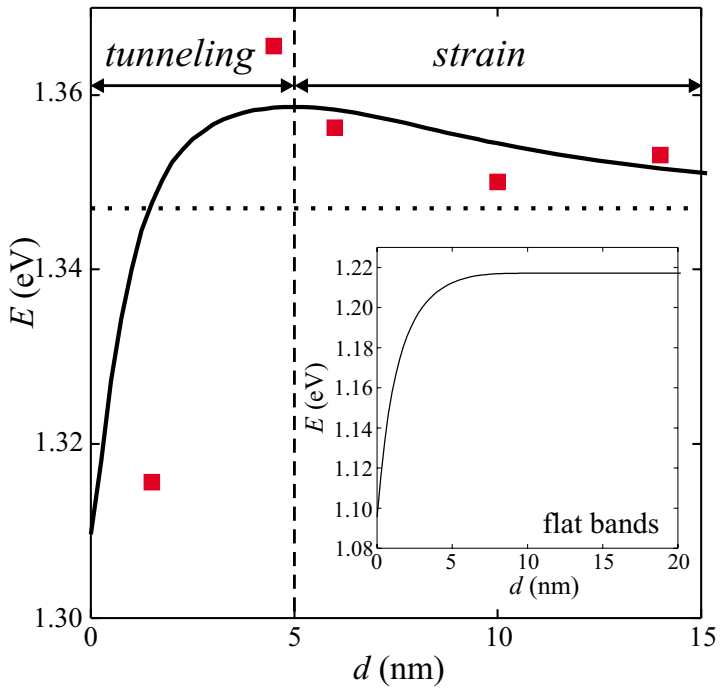

FIG. 4. (Color online) The variation in the ground $1 S^{+}$exciton energy with inter-ring separation (solid line). The experimental energies are shown by symbols. The energy level in the single quantum ring (dotted line) is close to the experimental energy, and the tunneling-dominated and strain-dominated regimes are indicated. The inset displays the similar dependence for the flat-band structure.

shown in Fig. 4 which exhibits a remarkable maximum around $d=d_{0}=5 \mathrm{~nm}$, and decreases slowly for $d>d_{0}$. The latter $d$ range is strain dominated, where tunneling is suppressed by strain effects. The exciton states are strongly influenced by tunnel coupling for $d<d_{0}$, and a rapid decrease in the exciton energy is found with decreasing $d$. We refer to this $d$ range as tunneling dominated. The ground exciton energy in the flat-band quantum-ring molecule is obviously fully in the tunneling-dominated regime, as shown in the inset of Fig. 4.

The blueshift of the exciton eigenenergy amounts to 11 $\mathrm{meV}$, and the slow convergence toward the exciton energy in a single quantum ring is mainly a consequence of the slow increase in the hole energy with $d$, as shown in Fig. 3. The experimental results of Ref. 10 are shown by the symbols in Fig. 4. One may notice that the experimental variation in the exciton energy on $d$ is rather well reproduced by the present model. In our calculation there are no fitting parameters and all input parameters are taken from the experiment. ${ }^{18}$ Therefore, we may conclude that we even obtain very reasonable quantitative agreement with experiment.

In conclusion, we found that nonuniform strain considerably modifies tunneling coupling in triple (In,Ga)As/GaAs quantum-ring molecules. The single-particle electron energies exhibit a remarkable crossing with increasing inter-ring separation, which may modify the optical absorption. On the other hand, because of the varying strain field with the interring distance, an almost steady increase in the ground hole eigenenergy with $d$ is found. Due to strain, both the electrons and holes in the ground state are mainly localized in the upper and lower rings. Peculiar variations in the singleparticle eigenenergies with inter-ring separation bring about the appearance of a blueshift of the exciton energy with respect to the result for a single quantum ring. Our theoretical results are compared with photoluminescence data, and good agreement is found without having to introduce any fitting parameters. We identified two regimes for the exciton states hybridization: strain dominated for $d>5 \mathrm{~nm}$ and tunneling dominated for $d<5 \mathrm{~nm}$.

This work was supported by the Flemish Science Foundation (FWO-V1), the EU NoE: SANDiE, the Belgian Science Policy (IAP), the Ministry of Science of Serbia, and the Brazilian Council for Research (CNPq).

\section{*tadic@etf.rs}

†rancois.peeters@ua.ac.be

${ }^{1}$ D. Loss and D. P. DiVincenzo, Phys. Rev. A 57, 120 (1998).

${ }^{2}$ G. Bester, J. Shumway, and A. Zunger, Phys. Rev. Lett. 93, 047401 (2004).

${ }^{3}$ G. Ortner, M. Bayer, Y. Lyanda-Geller, T. L. Reinecke, A. Kress, J. P. Reithmaier, and A. Forchel, Phys. Rev. Lett. 94, 157401 (2005).

${ }^{4}$ H. J. Krenner, M. Sabathil, E. C. Clark, A. Kress, D. Schuh, M. Bichler, G. Abstreiter, and J. J. Finley, Phys. Rev. Lett. 94, 057402 (2005).

${ }^{5}$ H. J. Krenner, E. C. Clark, T. Nakaoka, M. Bichler, C. Scheurer, G. Abstreiter, and J. J. Finley, Phys. Rev. Lett. 97, 076403 (2006).

${ }^{6}$ A. Lorke, R. J. Luyken, A. O. Govorov, J. P. Kotthaus, J. M. Garcia, and P. M. Petroff, Phys. Rev. Lett. 84, 2223 (2000).

${ }^{7}$ A. O. Govorov, S. E. Ulloa, K. Karrai, and R. J. Warburton, Phys. Rev. B 66, 081309(R) (2002).

${ }^{8}$ I. L. Kuskovsky, W. MacDonald, A. O. Govorov, L. Muroukh, X. Wei, M. C. Tamargo, M. Tadic, and F. M. Peeters, Phys. Rev. B 76, 035342 (2007).

${ }^{9}$ J. M. Garcia, G. Medeiros-Ribeiro, K. Schmidt, T. Ngo, J. L.
Feng, A. Lorke, J. Kotthaus, and P. M. Petroff, Appl. Phys. Lett. 71, 2014 (1997)

${ }^{10}$ D. Granados, J. M. Garcia, T. Ben, and S. I. Molina, Appl. Phys. Lett. 86, 071918 (2005).

${ }^{11}$ N. A. J. M. Kleemans, I. M. A. Bominaar-Silkens, V. M. Fomin, V. N. Gladilin, D. Granados, A. G. Taboada, J. M. Garcia, P. Offermans, U. Zeitler, P. C. M. Christianen, J. C. Maan, J. T. Devreese, and P. M. Koenraad, Phys. Rev. Lett. 99, 146808 (2007).

${ }^{12}$ L. K. Castelano, G. Q. Hai, B. Partoens, and F. M. Peeters, Phys. Rev. B 74, 045313 (2006).

${ }^{13}$ B. Szafran, F. M. Peeters, and S. Bednarek, Phys. Rev. B 75, 115303 (2007).

${ }^{14}$ M. Tadić, F. M. Peeters, K. L. Janssens, M. Korkusiński, and P. Hawrylak, J. Appl. Phys. 92, 5819 (2002).

${ }^{15}$ M. Tadić and F. M. Peeters, Phys. Rev. B 70, 195302 (2004).

${ }^{16}$ J. I. Climente, M. Korkusinski, G. Goldoni, and P. Hawrylak, Phys. Rev. B 78, 115323 (2008).

${ }^{17}$ Calvin Yi-Ping Chao and S. L. Chuang, Phys. Rev. B 46, 4110 (1992).

${ }^{18}$ I. Vurgaftman, J. R. Meyer, and L. R. Ram-Mohan, J. Appl. Phys. 89, 5815 (2001). 